\title{
Productos Forestales No Maderables de la comunidad El Tundo: un remanente boscoso de biodiversidad y conocimiento ancestral del sur del Ecuador Non-Timber Forest Products of the El Tundo community: a forested remnant of biodiversity and ancestral knowledge of southern Ecuador
}

Diana Maza Vivanco ${ }^{1 *}$, Sergio Abad², Omar Malagón ${ }^{1,3}$, Chabaco Armijos $^{1}$

DOI. 10.21931/RB/2021.06.04.5

Resumen: Los Productos Forestales No Maderables (PFNM) constituyen una fuente importante de subsistencia para las comunidades rurales del Ecuador. El presente estudio buscó identificar los PFNM más relevantes, así como su uso tradicional y actual en la comunidad El Tundo del cantón Sozoranga, Provincia de Loja. La información etnobotánica y etnozoológica se obtuvo a través 30 entrevistas orales y observaciones de campo. Se determinó el consenso entre los informantes en cada categoría de uso mediante (FIC), así como otros índices cuantitativos que permitieron calcular la importancia o valor cultural (IVU), validación científica (UST) y uso común (IF) de las especies. Se registraron 123 PFNM, de los cuales 116 fueron vegetales y 7 animales distribuidos en 12 categorías de uso, de las cuales destacaron la medicina humana y materiales. Las especies más versátiles localmente son Juglans neotropica Diels, Myroxylon peruiferum L. f, y Verbena litoralis Kunth. De las especies registradas, 33 constituyen recursos potenciales por su elevada aceptación socio-cultural, mientras que 17 de ellas presentaron los más altos niveles de fidelidad en toda la comunidad. Este trabajo contribuye a valorizar el conocimiento local para generar alternativas de aprovechamiento y uso sostenible de la biodiversidad.

Palabras clave: Biodiversidad, Productos Forestales No Maderables, El Tundo, Sur del Ecuador.

Abstract: Non-Timber Forest Products (NTFP) constitute an essential source of subsistence for rural communities of Ecuador. The present study identified NTFP and its traditional and current use in El Tundo community of Sozoranga canton, province of Loja. The ethnobotanical and ethnozoological information was obtained through 30 oral interviews and field observations. The consensus among informants in each category of use was determined using (FIC), as well as other quantitative indices that allowed calculating the importance or cultural value (IVU), scientific validation (UST), and everyday use (IF) of the species. 123 NTFP were registered, of which 116 were vegetables and 7 animals distributed in 12 categories of use, of which human medicine and materials stood out. The most locally versatile species are Juglans neotropica Diels, Myroxylon peruiferum L. f, and Verbena litoralis Kunth. Of the registered species, 33 constitute potential resources due to their high socio-cultural acceptance, while 17 present the highest levels of fidelity in the entire community. This work contributes to value local knowledge to generate alternatives for the sustainable use and harvesting of biodiversity.

Key words: Biodiversity, Non-Timber Forest Products, El Tundo, Southern Ecuador.

\section{Introducción}

En la actualidad existen varias definiciones para el término Producto Forestal No Maderable (PFNM), una de las más aceptadas es aquella propuesta por la Organización de las Naciones Unidas para la Agricultura y la Alimentación ${ }^{1}$ (1999) (FAO), quien los define como "bienes de origen biológico, distintos de la madera, derivados del bosque, de otras áreas forestales y de los árboles fuera de los bosques" (p.63). Existen muchas definiciones para este término que difieren unas de otras, existen dos aspectos fundamentales a la hora de considerar un PFNM, primero que sea dependiente de ecosistemas boscosos o terrenos análogos y segundo que sea excluyente de la madera².

En el Ecuador los PFNM han desempeñado un rol fundamental en la vida y el bienestar de los habitantes de diferentes sectores en cada una de las regiones naturales ${ }^{3}$, principalmente en la amazonia y la sierra ${ }^{4}$. En consecuencia, ha sido posible la instauración y el desarrollo de comunidades rurales campesinas e indígenas a lo largo del territorio ecuatoriano en particular en la región andina, en dónde la estrecha y longeva relación entre las diversas etnias que componen estas comunidades y el ecosiste- ma andino ha generado un amplio conocimiento sobre el uso de especies forestales en diversos ámbitos ${ }^{5-7}$ como alimentación $^{8-10}$, medicina ${ }^{11}$, tintes ${ }^{12}$, fibras y materiales de construcción ${ }^{13,14}$.

A pesar de la gran riqueza vegetal (3039 spp.) y animal (568 spp.) existente en la provincia de Loja ${ }^{15}$, la información sobre PFNM aún es escasa. Por lo tanto resulta imprescindible conocer la interacción que mantienen las comunidades con la biota animal y vegetal, con la finalidad de lograr un manejo sostenible de estos recursos para promover la conservación de los ecosistemas forestales, y en especial de los bosques montanos andinos que a pesar de ser considerados como uno de los puntos calientes de biodiversidad o "hotspots" a nivel global, han sido muy poco estudiados y los más amenazados en los trópicos ${ }^{16-18}$.

Es así que tomando en cuenta estas premisas, se decide dar inicio con un estudio etnobiológico en la comunidad rural El Tundo en la provincia de Loja, la cual por sus condiciones bióticas y climáticas forma parte de los bosques de neblina montanos del Ecuador de acuerdo a la clasificación de Sierra ${ }^{19}$, mientras que correspondiendo con el sistema de clasificación

\footnotetext{
Departamento de Química, Universidad Técnica Particular de Loja, Ecuador.

${ }^{2}$ Departamento de Obras Públicas, Gobierno Autónomo Descentralizado del cantón Sozoranga, Ecuador

${ }^{3}$ Proyecto PROAMAZONIA-UTPL, Universidad Técnica Particular de Loja, Ecuador.
} 
más reciente del $\mathrm{MAE}^{20}$, forma parte del Bosque siempreverde montano del Catamayo-Alamor (BsMn04). De todas maneras este ecosistema o formación vegetal constituye un sitio biodiverso con pocos remanentes naturales y en estado crítico en los Andes sur occidentales del país, por lo cual constituye un área natural prioritaria para la conservación ${ }^{20-22}$.

El objetivo del presente estudio fue valorizar el uso tradicional de los PFNM de la comunidad El Tundo del cantón Sozoranga en la provincia de Loja. A través de los resultados se podrán identificar potenciales PFNM y establecer acciones correctivas o generar alternativas que promuevan un uso sustentable de las especies forestales.

\section{Materiales y métodos}

\section{Localización}

El estudio se realizó en la comunidad rural El Tundo, situada dentro del Bosque Protector Jatumpamba-Jorupe en el cantón Sozoranga de la provincia de Loja, sur de Ecuador. Geográficamente se ubica entre las coordenadas geográficas $X$ : 632767 y Y: 9523280 , a una altitud de $1.800-2.400$ m s.n.m. (Figura 1).

\section{Recopilación de información}

Para esta fase se realizó previamente un Acta de Permiso de Consentimiento Previo Fundamentado, la cual permitió acceder al conocimiento ancestral ligado al uso tradicional de especies útiles. Se realizaron varios recorridos por todas las 18 viviendas que forman parte de esta comunidad. Se aplicaron 30 encuestas semiestructuradas correspondientes al 54\% de la población total, considerando personas de ambos sexos con rangos de edad entre 28-94 años.

Algunos de los datos considerados en la encuesta para especies vegetales y animales fueron los siguientes: nombre común, categoría de uso (alimentación, bebida, combustión, cuidado personal, forraje, material, medicina animal, medicina tradicional, medicina humana, miel de insectos, ornamental y tintura), parte utilizada, forma de recolección, modo de preparación y administración, manejo, y abundancia; mientras que para los informantes se consideró la edad, sexo, nivel de educación formal, actividad económica, tiempo de estancia en la comunidad, y tipo de vivienda.

\section{Recolección de muestras botánicas}

Durante varios recorridos de campo por zonas boscosas y de cultivo, se colectaron las muestras botánicas de las especies mencionadas por los informantes. Esta actividad se realizó con la participación y acompañamiento de un grupo de personas de la comunidad. Para la colecta, procesamiento y preparación del material vegetal se utilizó la guía propuesta por Cascante ${ }^{23}$.

\section{Identificación de especies de flora y fauna}

La determinación taxonómica de las muestras vegetales recolectadas se realizó en el ex Herbario de Plantas de Productos Naturales (HPPN), de la sección de química básica y aplicada del Departamento de Química de la Universidad Técnica Particular de Loja (UTPL), mediante la verificación con

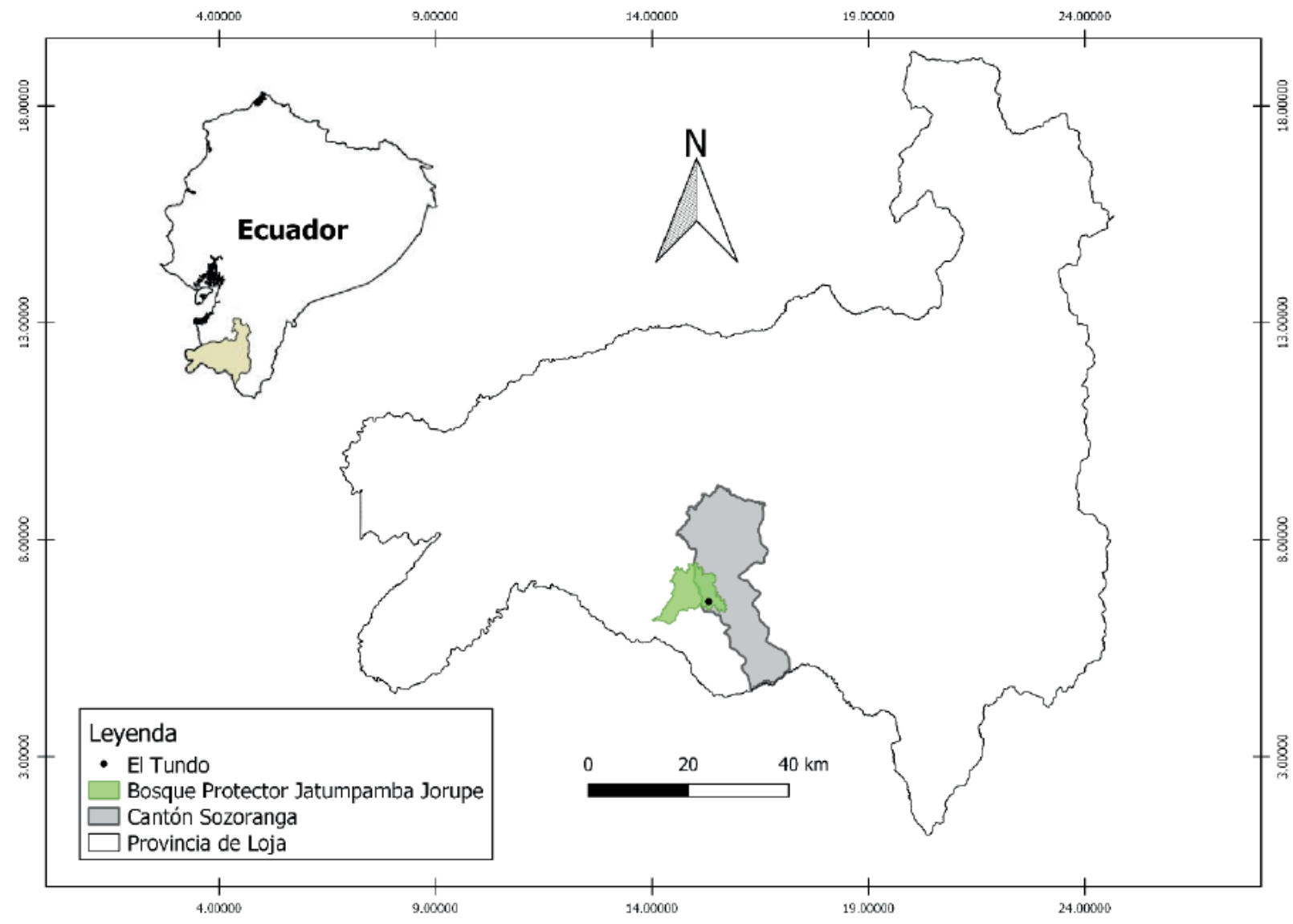

Figura 1. Posición geográfica del cantón Sozoranga y el área de estudio. 
pliegos de muestras botánicas ya existentes en dicho herbario. A su vez, se utilizó literatura complementaria de estudios etnobotánicos como la de Andrade et al. ${ }^{24}$; Tinitana Imaicela ${ }^{25} ; \mathrm{y}$ Tene et $a l^{26}$.

Para corroborar la información en cuanto a su taxonomía y origen biogeográfico de las especies vegetales, se consultó la base de datos on-line Tropicos del Missouri Botanical Garden ${ }^{27}$ (https://www.tropicos.org), el Catálogo de plantas vasculares del Ecuador ${ }^{28}$, y el Catálogo de la Vida ${ }^{29}$ (https://www. catalogueoflife.org); mientras que para las especies animales se consultó el portal de FaunaWebEcuador ${ }^{30}$ (https://bioweb. bio/faunaweb.html) y la base de datos de Global Biodiversity Information Facility (GBIF) 31 (https://www.gbif.org).

Para conocer el estado de conservación de las especies se revisó la Lista Roja de la Unión Internacional para la Conservación de Naturaleza ${ }^{32}$, el Libro Rojo de las plantas endémicas del Ecuador ${ }^{33}$, y el Libro Rojo de los mamíferos del Ecuador ${ }^{34}$. La nomenclatura taxonómica utilizada fue acorde a la propuesta por Jørgensen y León-Yánez ${ }^{28}$; Tirira ${ }^{35}$; y Tirira et al. ${ }^{36}$.

\section{Índices etnobotánicos cuantitativos calculados}

La información registrada a partir de las encuestas realizadas fue ingresada y organizada en una matriz de datos para su análisis cuantitativo. Para determinar los acuerdos (coincidencias) de los informantes sobre los PFNM utilizados en cada categoría de uso, se calculó el Factor de Consenso del Informante $(\mathrm{FIC})^{37,38}$, usando la siguiente fórmula:

$$
\mathrm{FIC}=\frac{\text { Nur }-\mathrm{Nt}}{\text { Nur }-1}
$$

Dónde:

Nur = número total de citaciones reportadas para una categoría de uso.

$\mathrm{Nt}=$ número total de especies utilizadas para esa categoría de uso.

De la misma forma se calculó el Índice de Valor de Uso (IVU), para determinar la importancia o valor cultural de una determinada especie para todos los informantes de la comunidad $^{39}$, empleando la siguiente fórmula:

$$
I V U=\frac{\sum U V}{n_{s}}
$$

Dónde:

UV = número de usos mencionados por cada uno de los informantes para cada especie.

ns = número de informantes encuestados.

Asimismo se calculó el Índice de Nivel de Uso Significativo TRAMIL (UST) para estimar en base a la frecuencia de usos ( $\geq$ 20\%), si una especie merece su evaluación y validación científica ${ }^{40}$, para ello se aplicó la siguiente fórmula:

\section{UST $=\frac{\text { Uso Especie }(\mathrm{s})}{\mathrm{n}_{\mathrm{s}}} * 100$}

Dónde:

Uso especie(s) = número de citaciones para cada especie. ns = número de informantes encuestados.
Finalmente para conocer la existencia de una posible especie con un mismo uso común en cada categoría de uso por toda la comunidad, se aplicó el Índice de Nivel de Fidelidad $(F L)^{41}$ utilizando la siguiente fórmula:

$$
\mathrm{FL}=\frac{\mathrm{Ip}}{\mathrm{It}} * 100
$$

Dónde:

I $\mathrm{p}$ = número de informantes que mencionan el uso de una especie para una categoría en particular.

It = número total de informantes que reportan esa especie para cualquier categoría.

\section{Resultados y discusión}

\section{Especies y familias reportadas}

En este estudio se reportaron 226 usos diferentes para 116 especies vegetales y 7 especies animales proveedoras de PFNM (Tabla 1). Estas especies en su mayoría crecen en zonas boscosas, mientras que otras son cultivadas en huertos agroforestales y jardines. Estos resultados guardan relación a los de Añazco ${ }^{42}$, quien inventarió en cuatro formaciones vegetales de la región andina ecuatoriana 100 PFNM, de los cuales fueron 90 especies vegetales y 10 especies animales. Asimismo, en otros estudios realizados anteriormente dentro de la provincia de Loja, se muestran cifras similares en número de especies vegetales reportadas como PFNM. En el cantón Zapotillo se han registrado 87 especies vegetales ${ }^{43}$, en el cantón Saraguro se reportan 89 spp. ${ }^{44}$, en el cantón Macará se enlistan 111 spp. ${ }^{45}$, y en el Parque Nacional Yacuri en el cantón Espíndola se registran 209 spp. ${ }^{4}$.

Las especies registradas se agruparon sistemáticamente en 54 familias y 95 géneros. Para las especies vegetales, las familias botánicas más representativas fueron Asteraceae (8 spp.), Poaceae (8 spp.), y Solanaceae (7 spp.). En cuanto a las especies animales, las familias registradas fueron Apidae, Dasypodidae, Didelphidae, Felidae, Mephitidae, Procyonidae, y Tayassuidae cada una con 1 especie. En diversos estudios etnomedicinales la familia Asteraceae se ha registrado como la más numerosa en especies ${ }^{46-48}$ y consecuentemente la más relevante en la comercialización de sus especies con fines terapéuticos entre la población ecuatoriana ${ }^{49}$. La gran cantidad de metabolitos secundarios ${ }^{50}$, y compuestos aromáticos con propiedades carminativos y desinfectantes ${ }^{51}$ que esta familia botánica produce puede estar relacionado con el valor medicinal que se le atribuye a la misma.

\section{Categorías de uso registradas}

Entre las diferentes categorías de uso reportadas, el mayor número de registros se obtuvo para (i) medicina humana (60 spp.), seguido del uso para (ii) elaboración de bebidas (39 spp.) y (iii) alimento (33 spp.). Es importante resaltar que una misma especie en algunos casos estuvo presente en más de una categoría de uso, lo cual demuestra la importancia de los usos o aplicaciones que tienen los recursos forestales para los pobladores de la comunidad El Tundo (Figura 2).

En Ecuador y particularmente en la provincia de Loja también se destaca y prevalece el uso medicinal como el más predominante y a su vez importante al igual que el uso de alimentación humana ${ }^{43-45,52-54}$. Estos reportes corroboran el uso habitual y constante de la medicina herbolaria en la población como una 

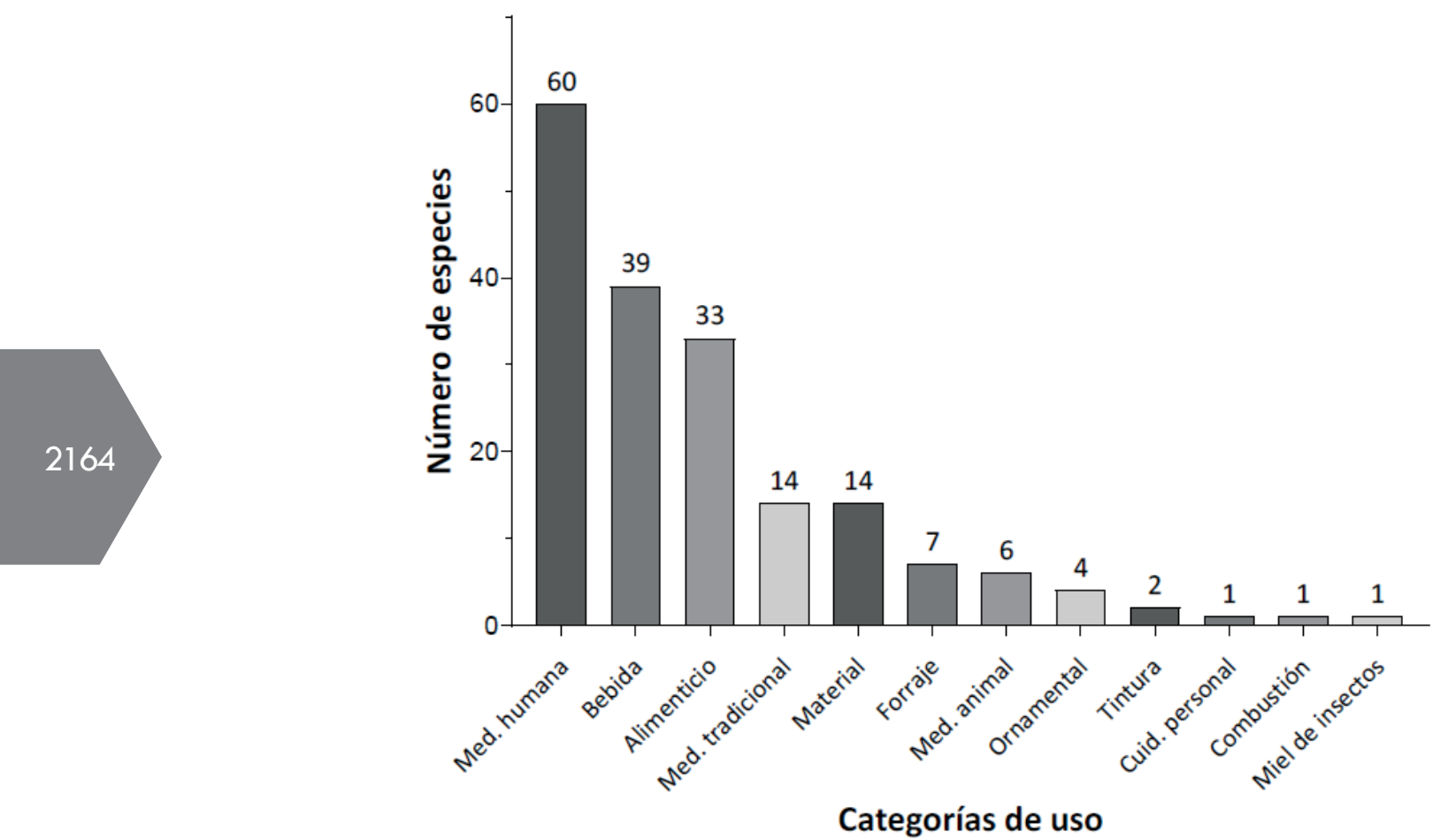

Figura 2. Número de especies reportadas como PFNM para cada categoría de uso en la comunidad El Tundo.

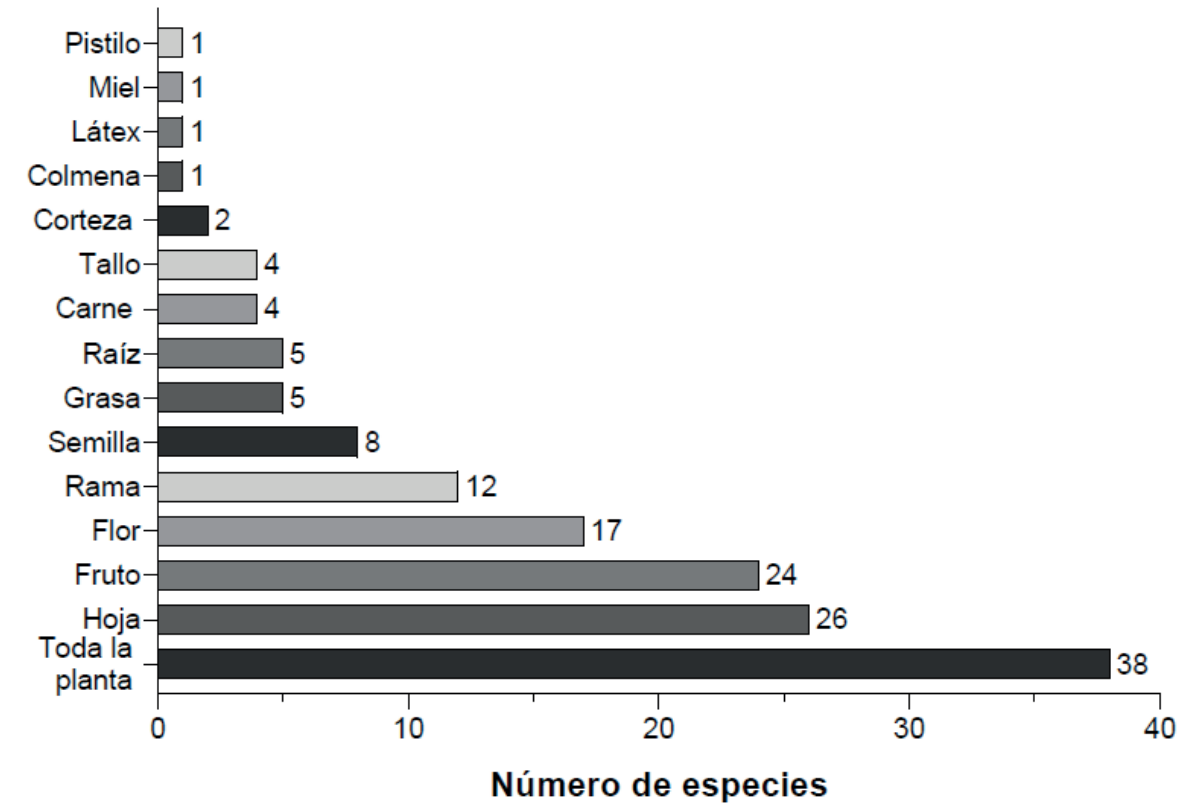

Figura 3. Estructuras útiles de las especies proveedoras de PFNM en la comunidad El Tundo.

alternativa natural de sanación. Por consiguiente se demuestra que el gran número de las especies que forman parte de estas categorías de uso ejercen un papel fundamental en la vida de los habitantes de la comunidad El Tundo, en especial aquellas relacionadas para atenuar problemas de salud puntuales.

Por otro lado la relación ser humano-fauna constituye también una importante interacción que las personas de esta comunidad han mantenido. Si bien, las especies de este grupo taxonómico reportadas en este estudio (7 spp.) no son tan numerosas en comparación a las especies vegetales (116 spp.), se han encontrado importantes usos para las mismas como son: (i) medicina humana (6 spp.), (ii) alimenticio (4 spp.), (iii) medicina animal (1 spp.), (iv) medicina tradicional (1 spp.), y (v) miel de insectos (1 spp.).
A nivel nacional se han realizado estudios etnozoológicos con fines de conservación, y registro de usos de PFNM en donde se incluyen las categorías de medicina humana y alimentos como las más habituales ${ }^{42}$, además de otras utilidades complementarias como ritual, animal de compañía ${ }^{55}$, y melífera ${ }^{56}$.

\section{Formas de vida, rango biogeográfico, y estado de conservación}

\section{de las especies}

Las 123 especies animales y vegetales se encuentran organizadas morfológicamente en ocho formas de vida. Las hierbas representan la mayor asignación con un total de (60 spp), seguido de los arbustos (27 spp.), árboles (23 spp.), mamíferos (6 spp.), trepadoras (2 spp.), e insectos, lianas, y subarbustos (1 spp.) cada uno. Del total de plantas vasculares documentadas 
en el Ecuador hasta el momento, las hierbas representan el hábito más numeroso en especies ${ }^{28}$ y en especies útiles para alguna categoría ocupa el segundo lugar después de los árboles ${ }^{53}$.

La relevancia del hábito herbáceo en términos de uso medicinal en la región sur del Ecuador también se ha documentado en diversos estudios previos $6,24,46,57,58$. Por otro lado el predominante uso de las hierbas, puede estar relacionado con el ecosistema boscoso montano del que forma parte el área de estudio, en dónde el estrato herbáceo es abundante ${ }^{20,22}$, y por la gran concentración de metabolitos secundarios que este grupo posee ${ }^{59}$. Esto refuerza la idea de la alta frecuencia del uso del hábito herbáceo por parte de la comunidad.

Del total de especies reportadas (123 spp.), se lograron identificar taxonómicamente hasta nivel de especie 109 spp., de las cuales 38 son nativas, 35 son introducidas y cultivadas, 13 son nativas y cultivadas, 10 son introducidas, 8 son cultivadas y 3 son endémicas; mientras que de las 2 restantes no se dispone de información con respecto a su origen en publicaciones especializadas.

De las 38 especies nativas, 22 de ellas tiene usos en medicina humana. En estudios realizados en las provincias de Loja y Zamora Chinchipe se muestran cifras superiores de especies nativas medicinales ${ }^{24,26,57,58}$. Estos datos posiblemente sean el resultado de la gran diversidad de especies vegetales útiles nativas identificadas en el Ecuador (4.591 spp.), cifra que representan el $89 \%$ de la totalidad de especies útiles registradas en el país ${ }^{53}$.

Sin embargo esta biodiversidad nativa se puede ver comprometida por la presencia de una gran cantidad de especies introducidas e introducidas y cultivadas en la zona, pues según Nuñez y Simberloff60 las especies exóticas o invasoras constituyen una seria amenaza para la persistencia de la diversidad. El extenso uso de plantas introducidas posiblemente sea consecuencia del gran valor alimenticio y medicinal que estas poseen.

Es así que en Ecuador especies introducidas como Coffea arabica L. (café), Saccharum officinarum L. (caña de azúcar), y Musa paradisiaca (banano) constituyen especies emblemáticas con fines alimenticios ${ }^{60}$ (Nuñez y Simberloff, 2005). Mientras que en el ámbito de la medicina, las especies introducidas destacan por su versatilidad para el tratamiento de diversas patologías, y por su amplia disponibilidad en todo el territorio nacional ${ }^{61}$. Lo cual reafirma su elevado grado de utilidad por parte de la población local.

Para la clasificación de acuerdo al estado de conservación, se consideraron las 109 especies animales y vegetales identificadas taxonómicamente hasta nivel de especie. Estas se encuentran distribuidas en seis categorías en función al sistema de clasificación de la UICN. La mayor parte de las especies (68 spp.) se encuentran en el primer nivel de clasificación, No Evaluado (NE), le sigue la categoría de Preocupación Menor (LC) con (28 spp.). Con menor número de especies están aquellas que presentan Datos Insuficientes (DD) (7 spp.), En Peligro (EN) se encuentran (3 spp.), Casi Amenazadas (NT) están (2 spp.), y en estado Vulnerable (VU) se encuentra (1 spp.)

De las ocho categorías, tres de ellas [En Peligro Crítico (CR), En Peligro (EN), y Vulnerable (VU)] implican amenaza ${ }^{62}$. En este contexto, cuatro especies de las identificadas se encuentran bajo algún grado de amenaza. En el caso de las especies vegetales, aquellas que se encuentran En Peligro (EN) son el café (Coffea arabica L.), el nogal (Juglans neotropica Diels.) y el monte del oso (Siparuna eggersii Hieron.) tanto en la Lista Roja de la UICN ${ }^{32}$ como en el Libro Rojo de las plantas endémicas del Ecuador ${ }^{33}$ para el caso de las dos últimas espe- cies. En cuanto a las especies animales, únicamente el león o puma (Puma concolor) se encuentra catalogado como Vulnerable (VU) a nivel global en la Lista Roja de la UICN ${ }^{32}$ y a nivel nacional en el Libro Rojo de los mamíferos del Ecuador ${ }^{34}$.

En términos de uso, los que más destacan para estas especies amenazadas son el uso medicinal y alimenticio. Ambas utilidades conllevan para el caso de las especies vegetales la recolección de partes aéreas de la planta, lo cual en modo constante genera la destrucción total de los individuos, mientras que para los animales implican la muerte inmediata del individuo. Probablemente estas circunstancias han inducido a nivel global a que buena parte de las especies medicinales principalmente (alrededor de 15.000), formen parte del listado de especies amenazadas registradas por la UICN63. A su vez factores de presión adicionales como la deforestación, fragmentación de hábitats, prácticas agroforestales inadecuadas, sobreexplotación de la flora y fauna silvestre, entre otros ponen en riesgo la perdurabilidad de los PFNM y por ende los medios de subsistencia especialmente de las familias de escasos recursos ${ }^{64-69}$.

El análisis de las cifras indica a su vez que cerca del 68\% de especies útiles para la comunidad, no poseen una evaluación en cuanto a su riesgo de extinción, lo cual las posiciona en las categorías de No Evaluado (NE) o Datos Insuficientes (DD); sin embargo esto no quiere decir que no se encuentren amenazadas $^{62}$. A nivel de la región, los estudios sobre el estado de conservación son escasos para los grupos taxonómicos de plantas medicinales ${ }^{46}$ e insectos tales como las abejas ${ }^{70}$. Es por ello crucial dar inicio con investigaciones que aporten información suficiente para esclarecer las probabilidades de extinción de estas importantes especies y poder establecer prioridades de conservación.

\section{Estructuras morfológicas y derivados utilizados de las especies}

Con respecto a las partes vegetales más aprovechadas, la planta entera presenta el mayor porcentaje de uso que reúne 38 spp. (31\%), seguido de las hojas con 26 spp. (21\%), y frutos con 24 spp. (20\%); menores proporciones tienen las flores que registran 17 spp. (14\%), ramas con 12 spp. (10\%), semillas con 8 spp. (6\%), raíces con 5 spp. (4\%), tallos con 4 spp. (3\%), cortezas con 2 spp. (2\%), por su parte el látex y los pistilos con 1 sola spp., representan el (1\%) cada una. Por otro lado en cuanto a los derivados de origen animal, la grasa (manteca) con 5 spp., y carne con $4 \mathrm{spp}$. se utilizan en mayores proporciones ( 4 y $3 \%$ respectivamente), mientras que la miel y colmenas con 1 spp. se emplean muy poco (1\%) (Figura 3).

Si bien, se ha identificado a las hojas y frutos como las formas vegetativas más utilizadas como PFNM en los bosques andinos ecuatorianos ${ }^{42}$; toda la estructura vegetal representa también una importante estructura con diferentes utilidades. En tal sentido en el presente estudio se incluyen 6 categorías de uso diferentes para las 38 especies de las cuales se aprovecha toda su estructura como materia prima. Entre las diferentes utilidades más frecuentes descritas para esta parte morfológica están el uso con fines medicinales, para elaboración de bebidas y para fabricación de herramientas domésticas.

Estos resultados guardan relación con lo reportado al sur del país, en la provincia de Loja por Bussmann y Sharon ${ }^{47}$; y Andrade et $a l^{24}$, quienes identifican a toda la planta como la estructura morfológica mayormente utilizada con fines medicinales, y Rios et al. ${ }^{46}$ a las hojas y flores como los órganos vegetales principalmente utilizados en la elaboración de bebidas

De todas maneras, las hojas al igual que las flores tam- 
bién forman parte de esta estructura útil (toda la planta) de las herbáceas frecuentemente reportada; por ende su uso habitual puede estar relacionado con las sustancias activas y compuestos químicos almacenados en forma de metabolitos secundarios que contienen las hojas principalmente de las hierbas medicinales ${ }^{71,72}$.

Para el caso de las partes y derivados de origen animal, se aprovecha la grasa, carne, y miel principalmente para la medicina humana y alimento. Es así que los animales silvestres constituyen para las comunidades rurales una fuente de calorías, proteínas y grasas esenciales ${ }^{73}$. Entre las especies mencionadas para el consumo de carne están: armadillo (Dasypus novemcinctus); león o puma (Puma concolor); sajino (Pecari tajacu); y shushano o coatí (Nasua narica). Aunque se esperaba que por la posición geográfica del área de estudio el impacto hacia la fauna silvestre sea aún más significativo, esto no fue así debido a que los pobladores de la comunidad emplean con mayor frecuencia fuentes de proteína alternativas procedentes de la crianza de animales domésticos.

Sin embargo el valor medicinal que se le atribuye a la grasa de los mamíferos es de gran importancia local, pues se registran beneficios curativos y preventivos que no lo poseen las plantas; por ejemplo para tratar manchas de la piel se emplea la raposa (Didelphis marsupialis), fracturas de huesos el shushano o coatí (Nasua narica), y combatir enfermedades reumáticas el añango (Conepatus semistriatus); shushano o coatí (Nasua narica), y respiratorias el armadillo (Dasypus novemcinctus). Todo esto se corrobora con los registros de usos similares para la grasa y carne de estos y otros mamíferos en Ecuador ${ }^{55,74}$, Colombia ${ }^{75,76}$ y México ${ }^{77-80}$. Cabe resaltar que para todas estas especies animales mencionadas existen criterios de selección por parte de los consumidores, es así que son capturados y aprovechados aquellos individuos más desarrollados que presenten mayor masa corporal. Esta particularidad puede conllevar a un desequilibro en la estructura poblacional de las especies, afectando así las posibilidades de desarrollo y reproducción de las mismas ${ }^{75}$.

Por otro lado el uso medicinal de la miel de abeja como PFNM se ha reportado también en la comunidad de Quimis en la provincia de Manabí, debido a sus propiedades curativas ${ }^{56}$. En este estudio se reporta el uso de la miel de abeja (Apis meLifera Linnaeus) para el restablecimiento de cortes y heridas, uso que está relacionado con la acción anti-bacteriana, anti-oxidante y anti-inflamatoria de los componentes de la miel para la reparación y cicatrización de tejidos ${ }^{81,82}$. Sin embargo, actualmente esta práctica de extracción de miel a partir de apiarios ya no se realiza entre los campesinos de la comunidad, por ende, se ha generado una pérdida del conocimiento tradicional en torno a esta práctica.

\section{Estado de uso o consumo de las especies}

El uso o consumo de los PFNM se distribuye en cinco estados, de los cuales algunas especies se emplean en más de un estado. En la mayor parte de los casos se aprovecha el material animal y/o vegetal de las especies en estado fresco (82 spp.), sin embargo, también se emplea bien sea fresco o seco (17 spp.), o por su parte únicamente en seco (13 spp.). Para el caso de frutos y algunas semillas comestibles, se consumen con más frecuencia en estadio maduro (26 spp.), y muy poco inmaduro (2 spp.).

En otros estudios realizados en la provincia de Loja, el uso de material vegetal fresco para preparar remedios y bebidas también es más común que en estado $\operatorname{seco}^{46,47,58}$. Asimismo el consumo de frutos y semillas es mayor en estado maduro ${ }^{83-85}$.
De acuerdo con Jima y Megersa ${ }^{86}$; Mata Pinzón et al. ${ }^{87}$, el uso de plantas frescas o recién cosechadas se debe a que las sustancias activas de las mismas se conservan en su mayoría en estado fresco, que por el contrario se perderían durante el transcurso de secado.

Por su parte el aprovechamiento frecuente de una gran parte de frutos en estado maduro puede atribuirse a las características organolépticas (textura, color, aroma, sabor, nivel de dulzura) que presentan los frutos en este estado de desarrollo, lo cual genera un aspecto atractivo para su aceptación y consumo 88,89 . Sin embargo por su estado de madurez, los frutos con esta característica se clasifican como productos perecibles, lo cual supone una tendencia a sufrir deterioro fisiológico y por ende de sus propiedades físico-químicas desde el momento de su cosecha ${ }^{90}$. Es así que por este motivo los informantes mencionan que el consumo o preparación (para algunos casos) de los frutos es inmediato. Sucede lo contrario con las semillas y granos ya que al ser consumidas en estado seco estas contienen bajos niveles de humedad, es decir menos del $50 \%$ de agua en su composición, a esto se suman sus propiedades antioxidantes lo cual a su vez favorece una larga preservación y más aún si se almacenan en lugares libres de humedad ${ }^{91,92}$. En la comunidad El Tundo las cosechas de gramíneas y leguminosas se conservan por largos periodos de tiempo en cajones de madera, ya que al ser una fuente rica de proteínas y carbohidratos ${ }^{93}$, estas forman parte de la dieta diaria de las familias de esta comunidad.

\section{Modo de consumo, empleo y preparación de las especies}

La forma de preparación o consumo de las especies animales y vegetales varía según la categoría de uso y parte utilizada. En este contexto, la infusión es la forma más usual de preparación de bebidas y remedios (53 spp.), seguida de la decocción (14 spp.), y con menor frecuencia están los atados, zumos, manojos, macerados, triturado, estrujado, licuado, frito, picado, molido, tostado, entero, lijado, partido, caliente y tejido.

En la comunidad El Tundo, el consumo de bebidas mediante infusión es una tradición. Dentro de estas bebidas se encuentra una de carácter medicinal, la cual es conocida localmente como "tisana". Dos de las formas más comunes para preparar una tisana son la infusión y la decocción $n^{94}$. En este caso, se trata de una infusión "bajeada" de 27 especies vegetales herbáceas y arbustivas frescas y/o secas, de las cuales se emplean partes suaves (hojas, tallos y ramas tiernas, y flores) para su preparación.

La preparación de estas partes tiernas de plantas medicinales mediante infusión, es el procedimiento más idóneo para la obtención de una buena tisana; debido a que se logra de una manera efectiva la extracción de los principios activos de las mismas, reduciendo la alteración de su estructura por acción del calor ${ }^{95}$. El consumo de esta infusión es habitual debido a sus efectos antiinflamatorios, además se le atribuye propiedades terapéuticas que tratan el "calor interno", y contrarrestan dolores renales.

En la provincia de Loja se ha registrado una bebida tradicional muy similar en aspecto y forma de preparación, comúnmente conocida como "horchata" que posee también propiedades antiinflamatorias ${ }^{46}$. El efecto antiinflamatorio en este tipo de bebidas puede estar relacionado con el proceso de preparación y con los compuestos químicos que poseen las plantas que forman parte de la fórmula de preparación. De acuerdo con Villegas-Novoa et al. ${ }^{96}$, Las infusiones herbales presentan diversos compuestos químicos que actúan en forma conjunta produciendo una respuesta antiinflamatoria. Entre 
Los compuestos químicos de las plantas medicinales García Bacallao et al. ${ }^{97}$ mencionan a los flavonoides y taninos, los cuales contribuyen a generar una respuesta antiinflamatoria.

A su vez dentro de esta categoría de infusiones se han mencionado otras propiedades terapéuticas por parte de los informantes como: analgésico, anticancerígeno (cáncer de la próstata), antigripal, infecciones del tracto urinario, sedante, hidratante, entre otras. Esta última es aprovechada mediante el consumo de "aguas aromáticas". Cabe mencionar que en algunos casos las infusiones se preparan mediante combinaciones, es decir se emplean dos o más especies; mientras que en otros casos se adicionan compuestos químicos como el bicarbonato de sodio, e incluso bebidas alcohólicas destiladas como el aguardiente de caña de azúcar. En un único caso en particular se registró el empleo de derivados de origen animal para preparación de infusiones medicinales; tal es el caso del nogal (Juglans neotropica Diels.), cuyas hojas se consumen en infusión con leche de vaca para producir un "aumento de la cantidad de sangre". Esta forma de preparación se ha documentado también en comunidades del Azuay y Cañar para tratar inflamaciones, afecciones bronquiales y anemia ${ }^{98}$.

\section{Forma de uso o administración de las especies}

Se han identificado 17 formas de uso o administración diferentes. Cabe mencionar que las formas de administración están relacionadas con aspectos referentes a la medicina, alimentación y bebidas; mientras que las formas de uso hacen énfasis a aquellas utilidades vinculadas de la obtención de un material o producto en especial. La administración vía oral fue significativamente la más frecuente (93 spp.). Otras formas de administración fueron baños y aplicaciones tópicas, inhalación, lavados y limpias, frotación, pomada, paños y emplasto, y soplos. Por otro lado, la forma de uso más frecuente fue como herramienta (11 spp.), y en menor proporción ornamento, construcción, tinte, calefacción, cocción, y cordelería. Una misma especie en algunos casos tiene más de una forma de uso o administración.

La forma oral, ha sido descrita también en varios estudios dentro de la provincia de Loja como la vía más frecuente de administración de remedios y bebidas ${ }^{47,57,58}$. Esto corrobora el empleo frecuente de la vía oral como principal mecanismo de administración de uso interno por parte de los usuarios de la comunidad, además de ser un medio de fácil y rápida asimilación de las propiedades medicinales ${ }^{71,99}$

Por su parte entre las formas de administración para uso externo están las limpias y los baños, las cuales se han registrado por parte de los informantes como aplicaciones (i) terapéuticas para el "resfrío de la sangre y de los huesos", "calor interno", "contrarrestar dolores de cabeza", "combatir resfriados", "endurar los bebés" y (ii) tradicionales para el "mal de aire" y "espanto de los niños". Estas vías de administración al igual que sus aplicaciones (terapéuticas y tradicionales) son de uso común en Loja y el resto de provincias que forman parte de los Andes ecuatorianos $11,26,98,100,101$.

A su vez las pomadas también forman parte de estas dos aplicaciones, principalmente para "aliviar hinchazones", "contrarrestar problemas reumáticos", "contrarrestar problemas de asma", "contrarrestar dolores de huesos y fracturas" y "mal de aire". Estos beneficios pueden estar relacionados con los efectos emolientes que posee esta forma de administración semisólida ${ }^{102}$.

Asimismo, las inhalaciones, frotaciones y soplos son formas adicionales de administración externa para tratar diferentes afecciones. En Ecuador, estas prácticas forman parte de la medicina ancestral andina ${ }^{7}$. Dentro de la comunidad El Tundo, se emplean principalmente en el campo de las aplicaciones tradicionales para tratar el "mal de aire" y el "espanto de los niños". En la comunidad de Saraguro de la provincia de Loja, los soplos por la boca corresponden a una práctica habitual para tratar enfermedades sobrenaturales tales como el "mal aire" o "susto"103. Mientras que en las comunidades rurales de México, las inhalaciones y frotaciones son métodos de administración externa frecuentes para atender el "mal de ojo" y el "susto"87.

Esto demuestra que estas formas de administración son sinérgicas para el tratamiento de este tipo de afecciones relacionadas con la medicina tradicional. La importancia que denotan las inhalaciones a la hora de contrarrestar el "mal de aire" puede atribuirse a los efectos que producen los aromas fuertes de las plantas utilizadas en el cuerpo de la persona afectada. Esta noción concuerda con lo reportado por (104) en su estudio etnobotánico con curanderos de la sierra ecuatoriana, quienes afirman que los olores fuertes de los órganos de las plantas generan una atracción intrínseca de "el mal" presente en el cuerpo humano. Tal es el caso de Ruta graveolens L, especie muy aromática ${ }^{100}$ que genera un efecto relajante en las personas posterior a su inhalación ${ }^{101}$. Sin embargo al tratarse de una especie con potencial tóxico, su uso debe ser controlado para evitar cuadros letales ${ }^{105}$.

Por otro lado, desde el punto de vista de los recursos materiales, el uso de los PFNM como herramientas ha desempañado un rol importante para cubrir ciertas necesidades de uso doméstico en las comunidades rurales del Ecuador ${ }^{14}$. Los objetos manufacturados que se han empleado por los informantes como herramientas son escobas, recipientes para transporte de agua, y productos tejidos denominados "shikras"; de los cuales estos dos últimos ya no se utilizan actualmente debido a que han sido sustituidos por utensilios plásticos o de otro material. Es así que el acceso al mercado es uno de los factores que promueve el desuso de las plantas como materia prima para confeccionar sus propios utensilios por parte de las comunidades ${ }^{106}$

De igual manera en el pasado se extraían tintes de color marrón de las hojas del nogal (Juglans neotropica Diels.) y se realizaban sogas a partir de las fibras foliares de cabuya (Furcraea andina Trel.), prácticas que actualmente han desaparecido entre los pobladores pero que son conocidas por muy pocos adultos mayores, quienes han estado durante toda su vida relacionados a este medio natural. Esto demuestra que la comunidad El Tundo ha sufrido un proceso de transculturización promovido por la no valorización de los conocimientos ancestrales, lo cual ha generado un abandono de estas prácticas que implican el desuso de especies clave en las culturas andinas. A nivel de los Andes ecuatorianos Juglans neotropica Diels., ha formado parte de las especies vegetales útiles para teñir lana ${ }^{107}$; mientras que Furcraea andina Trel. figuraba como uno de los principales PFNM más relevantes productores de fibra para la fabricación y comercialización de diversos utensilios ${ }^{108}$. Sin embargo, en los últimos años el uso tradicional de ambas especies ha sufrido un declive a causa de la globalización y disponibilidad de artículos industriales de menor precio $44,109,110$.

\section{Sistemas de manejo de las especies}

En cuanto al método de obtención o manejo de las especies que proporcionan PFNM, los cultivos representan la principal fuente de sustento para la comunidad (65 spp.), seguido del bosque (19 spp.), cultivos o pastizales (12 spp.), bosque o cultivos (10 spp.); un menor número de especies provienen de 
los jardines (8 spp), la caza (6 spp.), apicultura (1 spp.), compra (1 spp.), y de la compra o cultivos (1 spp.).

En la comunidad El Tundo es común el cultivo de plantas en huertos agroforestales y jardines por parte de hombres y mujeres, los cuales proveen alimentos y medicinas naturales que cubren sus requerimientos diarios. Estos sistemas productivos están compuestos por una diversidad de especies entre las que destacan especies base de su alimentación (hortalizas, tubérculos, cereales), árboles y arbustos frutales, hierbas medicinales, y especies avícolas y pecuarias. A nivel de la provincia de Loja, el cultivo en huertos agroforestales es una práctica habitual de las comunidades rurales ${ }^{111}$. La instauración de estos sistemas diversificados de producción especialmente en los sectores rurales tiene como finalidad garantizar la subsistencia de las familias campesinas ${ }^{112}$, y a su vez contribuir a la conservación in situ de la biodiversidad ${ }^{113}$. Esto ratifica la importancia que representan los cultivos agroforestales para esta comunidad.

Por otro lado, el bosque corresponde a una fuente silvestre de cosecha de PFNM, de donde los pobladores mencionan extraer inusualmente pocos productos únicamente para consumo propio. Esta tendencia es similar principalmente para especies medicinales, las cuales de acuerdo a los estudios de Ansaloni et $a l^{6}{ }^{6}$ y Solano Rivera ${ }^{57}$, se extraen muy poco del cerro o bosque debido a que son cultivadas en huertos y jardines. El hecho de que los pobladores de la comunidad no obtengan beneficios económicos de la venta de productos forestales medicinales, reduce en parte el impacto hacia este ecosistema. Pues se ha determinado que las cosechas y capturas masivas en los bosques se ven influenciadas por la demanda y el acceso a los mercados ${ }^{114}$. Cabe resaltar que el bosque en la comunidad El Tundo se encuentra en buen estado de conservación en las partes altas con pendientes fuertes que hacen el acceso limitado, mientras que en las zonas bajas y en parte planas la cobertura forestal se encuentra fragmentada o intervenida a causa del establecimiento de cultivos, pastizales y la deforestación, generando así un mosaico paisajístico en el lugar.

En cuanto a los PFNM de origen animal, estos se obtienen de la caza de especies silvestres. La cacería es una actividad bastante conocida en las comunidades rurales para la obtención de PFNM tales como carne y pieles ${ }^{115}$. Esta práctica es exclusivamente masculina, y a su vez poco frecuente entre los pobladores de esta comunidad, pues mencionan que los pocos ejemplares cazados son el resultado de una mera casualidad, y que además últimamente no se han observado animales silvestres por el sector y cuando aparecen se presentan muy pocos individuos, mientras que en otros casos algunas especies como el armadillo (Dasypus novemcinctus) han desaparecido por completo de la zona. Esto puede ser el resultado de la influencia de esta práctica sobre las estructuras poblaciones de las especies silvestres.

Al respecto Bennett y Robinson ${ }^{116}$, afirman que algunos de los efectos significativos de la caza en las poblaciones silvestres son la reducción de la densidad de las poblaciones y la extinción local de especies vulnerables. Lo cual se corrobora con el registro de una reducción poblacional de mamíferos silvestres a causa de la cacería como medio de subsistencia en las comunidades indígenas de los Sionas y Secoyas en el Ecuador $^{117}$. Otra causal pude atribuirse a la intervención antrópica que ha sufrido el bosque en la comunidad, lo cual ha generado la migración de las especies dependientes del bosque hacia otros remanentes boscosos ${ }^{118}$. De todas maneras la comprensión del manejo de los recursos de vida silvestre, sean plantas o animales por parte de los miembros de un pueblo o comunidad, resulta un hecho determinante para fomentar la conservación del bosque ${ }^{119}$.

\section{Factor de Consenso del Informante (FIC)}

En función de la utilidad dada por parte de los informantes, Los PFNM han sido clasificados dentro de 12 categorías de uso. Los factores de consenso del informante se han calculado para cada categoría de uso (Tabla 2). El valor más alto de FIC (0.78) se obtuvo para la categoría de medicina humana, mientras que, para las categorías de cuidado personal, combustión y miel de insectos, los valores fueron nulos (0.00), debido al reducido número de citaciones para una sola especie en estas categorías.

Los valores de FIC obtenidos muestran que existe un consenso entre los pobladores de la comunidad El Tundo respecto al uso de los PFNM en todas las categorías de uso a excepción de las tres últimas. Para el caso de las nueve primeras categorías de uso se evidencia que existe un intercambio de conocimientos y criterio de selección bien definido entre la comunidad. Estos resultados guardan relación a lo registrado por Briceño Fonseca et $a l^{120}$, quienes señalaron a las categorías alimenticia y medicinal como las de mayor valor de FIC (0.67 y 0.66 respectivamente) en cuanto al uso tradicional de PFNM.

Para el caso de las categorías de cuidado personal, combustión y miel de insectos, no se registró un consenso entre los informantes con respecto al uso específico de las especies registradas en estas categorías. Este escaso consenso podría ser el resultado de un proceso de transculturación o deterioro de la trasmisión del conocimiento de generación en generación, lo cual ha generado una erosión de conocimiento tradicional sobre el uso de las especies en dichas categorías en esta comunidad ${ }^{121,122}$. Esta idea se ve reforzada por el hecho de que especies como el nogal (Juglans neotropica Diels) y la abeja (Apis mellifera Linnaeus), actualmente ya no se emplean por los pobladores para el oscurecimiento del cabello y extracción de miel de abeja respectivamente, puesto que el acceso a productos comerciales ha provocado la pérdida de dependencia de estos PFNM.

Dentro de la categoría de medicina humana se encuentran subcategorías de uso, entre las más comunes y responsables del alto consenso (24 especies y 152 citaciones) para esta categoría están la influenza (gripe) y los resfriados. Este elevado acuerdo entre los informantes demuestra que ambas afecciones son comunes dentro de la comunidad, las cuales posiblemente aparecen como consecuencia de las condiciones climáticas (bajas temperaturas) propias de la zona; por lo cual a su vez se refleja la importancia que tienen las plantas medicinales en la localidad. Esto se corrobora con lo reportado por Tinitana et $a L^{58}$ y Rios et $a L_{.}{ }^{46}$, quienes registraron un elevado consenso ( $\mathrm{FIC}=0.91$ y 0.83 respectivamente) entre mujeres expendedoras de plantas medicinales para tratar resfriados y la influenza en mercados tradicionales de la provincia de Loja. Dichas afecciones forma parte de las enfermedades del sistema respiratorio, y constituye una de las principales causas de mortalidad a nivel nacional ${ }^{123}$. Por su parte Ullah y $\mathrm{Mu}$ hammad ${ }^{124}$, identificaron a los resfriados como la categoría de afecciones medicinales con mayor consenso $(F I C=0.98)$ entre los informantes del valle de Shamozai, norte de Pakistán, dónde el clima es frío en temporada invernal.

\section{Índice de valor de uso (IVU)}

De las 123 especies registradas, únicamente 9 de ellas presentaron los valores de uso más altos (IVU= $0.57-1.47)$. 
Esto puede atribuirse a la pérdida progresiva del conocimiento ancestral o interés de la población local en cuanto al uso de PFNM. Las especies que denotan mayor importancia o valor cultural para los usuarios de la comunidad El Tundo son aquellas de origen vegetal. Entre las que destacan están el nogal (Juglans neotropica Diels; 1.47), chaquino (Myroxylon peruiferum L. f; 0.90), verbena (Verbena litoralis Kunth; 0.77), manzanilla (Matricaria chamomilla L; 0.70), guineo (Musa sapientum L; 0.60), sauco (Cestrum peruvianum Willd. ex Roem. \& Schult; 0.60), malva olorosa (Pelargonium odoratissimum (L.) L'Her; 0.57), tilo (Sambucus nigra L; 0.57), y toronjil (Melissa officinalis L; 0.57) (Tabla 3). El resto de especies presentaron valores de uso por debajo de 0.50 .

Debido a la composición de aminoácidos presentes en $\mathrm{J}$. neotropica Diels, esta especie constituye una buena fuente proteica para la nutrición humana ${ }^{125}$. Así mismo por sus propiedades tónicas en el pasado varias mujeres de la comunidad elaboraban un "jarabe de alimento" a partir las hojas del nogal para tratar diferentes afecciones de salud, el cual era comercializado en plazas de la ciudad de Loja. Sin embargo en la actualidad esta práctica ya no se realiza, ya que su proceso de elaboración es extenso y además conocido por muy pocas personas dentro de la comunidad. Esta y otras prácticas ancestrales de la provincia de Loja, han sido documentadas para J. neotropica Diels en los estudios previos $24,26,53,98,125$, lo cual demuestra el valor potencial que ha tenido principalmente en el ámbito medicinal y la importancia que aún posee dicha especie para los pobladores locales.

Dentro de la provincia de Loja el nogal (J. neotropica Diels.) también ha sido identificado como uno de los PFNM con mayor valor de uso. Tanto en las comunidades de Bellavista, Cequer, El Durazno, San Antonio de Manú, y Udushi de la parroquia Manú del cantón Saraguro ${ }^{44}$, como en las comunidades de la parroquia Santa Rufina del cantón Chaguarpamba ${ }^{126}$, esta especie registra un (IVU=2) con utilidades en el ámbito de la medicina humana para el tratamiento de diversas afecciones medicinales, y para la obtención de tintes y colorantes. Estos registros ponen de manifiesto la importancia de dicha especie a nivel provincial.

Las múltiples utilidades que presentan las especies anteriormente mencionadas para los informantes ${ }^{127}$, así como su abundancia en la zona (en el caso de J. neotropica Diels.) ${ }^{128}$, han sido determinantes para su posicionamiento como las de mayor relevancia para la comunidad El Tundo. En contraste, las especies registradas con un menor IVU $(\leq 0.50)$, tienen en la mayoría de los casos un solo uso específico, como aquellas de uso alimenticio, material, y ornamental. Esta situación puede deberse a que varias de estas especies están entrando en desuso o su conocimiento es restringido por muy pocas personas de la comunidad, por lo cual su importancia o valor cultural no es significativo.

\section{Nivel de uso significativo TRAMIL (UST)}

Mediante el nivel de uso significativo, se registraron 33 especies vegetales con valores de UST iguales o superiores al 20\%. La especie con mayor UST el nogal (Juglans neotropica Diels) con $83.33 \%$, y las otras 32 especies tienen valores de UST entre $20 \%$ y $63.33 \%$ (Tabla 3 ).

Los valores de UST obtenidos muestran una elevada aceptación social y cultural por parte de la comunidad El Tundo principalmente para las especies introducidas (15 spp.), en comparación a las nativas (14 spp.) y cultivadas (2 spp.); a pesar de que las especies con origen biogeográfico nativo han sido registradas como las más abundantes en este estudio.
Posiblemente esto puede estar relacionado con la categoría de uso designada para estas especies introducidas, las cuales en su mayoría han sido nombradas por constituir una fuente de alimento, y soluciones rápidas de curación de afecciones relacionadas con la medicina animal, tradicional y humana.

Esto se corrobora con lo mencionado por Bennett y Pran$\mathrm{ce}^{129}$ y Begossi et al. ${ }^{130}$, quienes afirman que el predominio de la flora introducida es muy común principalmente para el uso alimenticio, medicinal, y ornamental en países tropicales, de los cuales Ecuador no es la excepción. El nivel de uso significativo que poseen estas plantas introducidas, se debe en gran parte a su uso frecuente por parte de los pobladores locales, lo cual se ve reflejado en el número de citaciones obtenidas para cada especie. Las 15 especies introducidas registradas entre las 33 con los porcentajes de UST $=\geq 20 \%$ son: Musa sapientum L. (56.67\%; 15 citaciones), Matricaria chamomilla L. (56.67\%; 17 citaciones), Pelargonium odoratissimum (L.) L'Her (43.33\%; 12 citaciones), Citrus x aurantium L. (36.67\%; 11 citaciones), Phyla dulcis (Trevir.) Moldenke (30\%; 9 citaciones), Cymbopogon citratus (DC.) Stapf (30\%; 9 citaciones), Coffea arabica L. (26.67\%; 8 citaciones), Rosa $\times$ alba L. (26.67\%; 8 citaciones), Ruta graveolens L. (26.67\%; 8 citaciones), Zea mays L. (23.33\%; 7 citaciones), Saccharum officinarum L. (20\%; 6 citaciones), Mentha piperita L. (20\%; 6 citaciones), Eriobotrya japonica (Thunb.) Lindl. (20\%; 6 citaciones), Phyla scaberrima (A. Juss. ex Pers.) Moldenke (20\%; 6 citaciones), y Aloe vera (L.) Burm.f. (20\%; 6 citaciones). La importancia de algunas de estas plantas no nativas está bien definida en el país, pues $C . x$ aurantium L., C. citratus (DC.) Stapf; y S. officinarum L. forman parte de la farmacopea indígena del Ecuador ${ }^{129}$.

En diferentes estudios etnomedicinales realizados a nivel nacional, algunos autores también han identificado varias de las especies anteriormente mencionadas con altos porcentajes de aceptabilidad social y cultural ${ }^{99,131-134}$. Estos registros confirman la alta preponderancia y versatilidad de dichas especies introducidas a nivel regional y nacional, estableciéndose como recursos vegetales importantes con posibilidades de ser objetos de estudio en futuras investigaciones orientadas a producir medicamentos o productos herbolarios a partir de ellas y de esta manera conseguir un aprovechamiento y uso de los compuestos activos que estas poseen.

\section{Índice de Nivel de Fidelidad (FL)}

Mediante el índice de nivel de fidelidad, se identificaron para las categorías de uso un total de 17 PFNM potenciales en términos de uso para la población local de El Tundo (Tabla 6). Para este propósito se consideraron las especies con un total de al menos 9 citaciones entre categorías de uso $(\mathrm{I}=9)$ y altos niveles de fidelidad. Cabe señalar que para las categorías de combustión, miel de insectos, y ornamental no se registraron especies, puesto que presentan citaciones inferiores a $9(\mathrm{It}=1)$.

Un FL del 100\% indica un elevado acuerdo entre la mayoría de los informantes de la comunidad El Tundo con respecto a su uso para una misma categoría. Un total de 8 especies vegetales presentaron un $\mathrm{FL}=100 \%$ (Verbena litoralis Kunth, Gynoxys verrucosa Wedd., Phyla dulcis (Trevir.) Moldenke, Myroxylon peruiferum L. f., Peperomia congona Sodiro, Cestrum racemosum Ruiz \& Pav, y Sambucus nigra L.); sin embargo, una de ellas no se encuentra identificada taxonómicamente por lo cual no se considerará para su análisis con otros estudios.

El uso de recursos vegetales para la elaboración de productos manufacturados tales como las escobas está claramente documentado en el Ecuador ${ }^{13}$, en dónde $V$. Litoralis Kun- 
th forma parte de las 10 especies más útiles tanto en el ámbito medicinal y como material ${ }^{10}$. En el presente estudio para la categoría de materiales, $V$. Litoralis Kunth destaca por su uso para la fabricación de escobas de uso doméstico. El empleo de esta y otras especies para esta finalidad también ha sido registrado en las comunidades de Chaguarpamba, Puyango, Paltas, Catamayo y Sozoranga ${ }^{125}$. Lo cual demuestra que, en las comunidades rurales y campesinas de la provincia de Loja, aún prevalece el uso de plantas como V. Litoralis Kunth para ser utilizadas como materia prima para la elaboración de escobas artesanales que por el contrario deberían ser adquiridas con dinero efectivo en los mercados.

La medicina tradicional incluye varias enfermedades de carácter espiritual o sobrenatural. El espanto forma parte de una de estas afecciones, la cual afecta principalmente a niños por tratarse de un grupo susceptible ${ }^{100}$, y su tratamiento requiere plantas medicinales como agentes terapéuticos ${ }^{87}$. En la comunidad El Tundo G. verrucosa Wedd es utilizada constantemente mediante baños o limpias corporales para contrarrestar el "espanto" de los niños. G. verrucosa Wedd es una especie de la familia botánica Asteraceae ampliamente utilizada dentro de la medicina tradicional en la provincia de Loja, a la cual se le atribuyen múltiples propiedades curativas para tratar la alergia, dermatitis, y el "espanto"25,26,53.

Las 5 especies con mayores valores de fidelidad registradas en la categoría de medicina humana se usan para contrarrestar dolores de cabeza $(M$. peruiferum L. f., $P$. congona Sodiro, y $C$. racemosum Ruiz \& Pav), dolores estomacales ( $P$. dulcis (Trevir.) Moldenke), cortes y heridas (M. peruiferum L. f.), prevención de los nervios y dolores de oído ( $P$. congona Sodiro), como antigripal (M. peruiferum L. f., P. congona Sodiro, C. racemosum Ruiz \& Pav, y S. nigra L.), anticancerígeno y energizante (M. peruiferum L. f.). Lo anterior coincide con varios autores quienes mencionan a estas especies para tratar los mismos problemas de salud en la provincia de Loja $24,25,47,56,134$.

Tanto la bibliografía científica como los elevados niveles de fidelidad obtenidos para Verbena litoralis Kunth, Gynoxys verrucosa Wedd., Phyla dulcis (Trevir.) Moldenke, Myroxylon peruiferum L. f., Peperomia congona Sodiro, Cestrum racemosum Ruiz \& Pav, y Sambucus nigra L., confirman la efectividad gracias a sus componentes activos y sobre todo demuestran un acuerdo considerable y un íntimo conocimiento entre los informantes de las comunidades rurales de la provincia de Loja, entre ellas la comunidad de El Tundo para el uso medicinal de estas especies.

A su vez los elevados niveles de fidelidad sugieren que estas especies están incorporadas dentro de las actividades diarias de los usuarios y por lo tanto se consideran habituales en la zona de estudio. Entre estas especies Myroxylon peruiferum L. f., debería ser considerado objeto prioritario en cuanto a conservación y manejo sostenible. Puesto que al ser una especie tan aceptada por sus múltiples propiedades medicinales, la extracción constante de su corteza conlleva a la destrucción definitiva de estos individuos arbóreos, y más aun teniendo en cuenta que es una especie especialista con distribución restringida en la provincia de Loja, lo cual aumenta sus posibilidades de extinción ${ }^{136}$

\section{Conclusiones}

El presente trabajo constituye uno de los primeros estudios etnobiológicos que incluye plantas y animales desarrollados en el sur del Ecuador. A su vez demuestra la estrecha relación que mantienen los pobladores de la comunidad El Tundo con la flora y fauna forestal, así como el amplio conocimiento tradicional adquirido y que todavía prevalece entre las personas adultas y adultas mayores de la localidad. Todo esto queda reflejado con las 123 especies proveedoras de PFNM registradas, las cuales han sido y son una fuente natural e indispensable principalmente para las categorías de medicina humana, alimentos y bebidas en esta comunidad.

\section{Agradecimientos}

Los autores agradecen a la Universidad Técnica Particular de Loja y al Gobierno Autónomo Descentralizado del Cantón Sozoranga por el apoyo y contacto con la comunidad.

\section{Financiamiento}

El proyecto fue financiado por el Fondo Mundial para el Medio Ambiente (GEF) y el Fondo Verde del Clima (GCF). El GEF otorga donaciones a países que inviertan en proyectos medioambientales mundiales en sostén del nexo vital entre agricultura y medioambiente, esto incluye cambio climático, biodiversidad, degradación de la tierra, aguas internacionales y productos químicos. El GCF se estableció como el mecanismo de financiamiento de la Convención Marco de las Naciones Unidas sobre Cambio Climático (CMNUCC) en 2010, encargado de apoyar a los países en desarrollo y en transición a medida que amplían sus esfuerzos para mantener el aumento de la temperatura global por debajo de $2^{\circ}$ Celsius, bajo el marco del Programa Integral Amazónico de Conservación de Bosques y Producción Sostenible (PROAmazonía).

\section{Conflicto de intereses}

Los autores declaran no tener conflicto de intereses.

\section{Referencias bibliográficas}

1. Food and Agriculture Organization of the United Nations. Towards a harmonized definition of non-wood forest products. In: Dember S., Perlis A (eds). Non-wood Forest Products and Income Generation. Unasylva, 1999.

2. Wong J, Thornber K, Baker N. Evaluación de los recursos de productos forestales no madereros. Organizacion de las Naciones Unidas para la Agricultura y la Alimentacion: Roma, 2001

3. Añazco M, Morales M, Palacios W, Vega E, Cuesta A. Sector Forestal Ecuatoriano: propuestas para una gestión forestal sostenible. Serie Inve. Quito -Ecuador, 2010.

4. Carrión JC, Hurtado S, Ulloa L, Herrera C. Productos forestales no maderables (PFNM) de la zona de amortiguamiento del Parque Nacional Yacuri, Espíndola, Loja, Ecuador. Bosques Latitud Cero 2019; 9: 83-93.

5. de la Torre L, Muriel P, Balslev H. Etnobotánica en los Andes del Ecuador. In: Morales R. M, Øllgaard B, Kvist LP, Borchsenius F, Balslev H (eds). Botánica Económica de los Andes Centrales. Universidad Mayor de San Andrés: La Paz-Bolivia, 2006, pp 246-267.

6. Ansaloni R, Wilches I, León F, Orellana A, Tobar V, Witte P De et al. Estudio Preliminar sobre Plantas Medicinales Utilizadas en Algunas Comunidades de las Provincias de Azuay , Cañar y Loja , para Afecciones del Aparato Gastrointestinal. Revista Tecnológica ESPOL 2010; 23: 89-97.

7. Orellana A, Achig D, Angulo A, Barrera G, Brito L, Mosquera L. Sabiduría Ancestral Andina y Uso de Plantas Medicinales. Principios y Prácticas de la Medicina Tradicional en Ecuador. Talleres Gráficos de la Universidad de Cuenca: Cuenca-Ecuador, 2020.

8. Ulloa C. Aromas y sabores andinos. In: Morales R. M, Øllgaard B, Kvist LP Borchsenius F, Balslev H (eds). Botanica Economica de los Andes Centrales. Universidad Mayor de San Andrés: La Paz-Bolivia, 2006, pp 313-328. 
9. Asturizaga AS, Øllgaard B, Balslev H. Frutos comestibles. In: Morales M, Øllgaard B, Kvist LP, Borchsenius F, Balslev H (eds). Botánica Económica de los Andes Centrales. Universidad Mayor de San Andrés: La Paz-Bolivia, 2006, pp 329-346.

10. Van den Eynden V, Cueva E. Las plantas en la alimentación. In: de la Torre L, Navarrete H, Muriel P, Macía MJ, Balslev H (eds). Enciclopedia de las Plantas Útiles del Ecuador. Herbario QCA de la Escuela de Ciencias Biológicas de la Pontificia Universidad Católica del Ecuador \& Herbario AAU del Departamento de Ciencias Biológicas de la Universidad de Aarhus, 2008, pp 62-66.

11. Cerón Martínez CE. Plantas medicinales de los Andes ecuatorianos. In: Morales M, Øllgaard B, Kvist L, Borchsenius F, Balslev H (eds). Botánica Económica de los Andes Centrales. Universidad Mayor de San Andrés: La Paz-Bolivia, 2006, pp 285-293.

12. Miller LM, Meisch LA. Weaving and Dyeing in Highland Ecuador. 1st ed. University of Texas Press: United States of America, 2007.

13. Macía MJ. Las plantas de fibra. In: Morales R. M, Øllgaard B, Kvist LP, Borchsenius F, Balslev H (eds). Botánica económica de los Andes Centrales. Universidad Mayor de San Andrés: La Paz-Bolivia, 2006, pp 370-384.

14. Macía M, de la Torre L. Los usos de las plantas para la obtención de materiales. In: de la Torre L, Navarrete H, Muriel P, Macía M, Balslev H (eds). Enciclopedia de las Plantas Útiles del Ecuador. Herbario QCA de la Escuela de Ciencias Biológicas de la Pontificia Universidad Católica del Ecuador \& Herbario AAU del Departamento de Ciencias Biológicas de la Universidad de Aarhus, 2008, pp 86-93.

15. Aguirre Mendoza Z, Aguirre Mendoza N, Muñoz Ch. J. Biodiversidad de la provincia de Loja, Ecuador. Arnaldoa 2017: 24: 523-542.

16. Myers N, Mittermeier RA, Mittermeier CG, da Fonseca GAB, Kent J. Biodiversity hotspots for conservation priorities. Nature 2000; 403: 853-858.

17. Bussmann RW. Bosques andinos del sur de Ecuador, clasificación, regeneración y uso. Revista Peruana de Biologia 2005; 12: 203216

18. Tapia-Armijos MF, Homeier J, Espinosa Cl, Leuschner C, de La Cruz M. Deforestation and forest Fragmentation in South Ecuador since the 1970s - Losing a Hotspot of Biodiversity. PLoS ONE 2015; 10: 1-18.

19. Sierra M. R. Propuesta preliminar de un sistema de clasificación de vegetación para el Ecuador continental. Proyecto INEFAN/ GEF-BIRF y EcoCiencia. Editorial Rimana: Quito-Ecuador, 1999.

20. MinisteriodelAmbientedelEcuador. Sistema de Clasificación de los Ecosistemas del Ecuador Continental. Subsecretaría de Patrimonio Natural: Quito-Ecuador, 2013.

21. 21. Lozano C. PE. Los tipos de bosque en el sur del Ecuador. In: Aguirre Z, Madsen J, Cotton E, Balslev H (eds). Botánica Austroecuatoriana: Estudios sobre los recursos vegetales en las provincias de El Oro, Loja y Zamora-Chinchipe. Herbario Reinaldo Espinosa: Loja-Ecuador, 2002, pp 29-49.

22. Tejedor Garavito N, Álvarez E, Arango Caro S, Araujo Murakami A, Blundo C, Boza Espinoza TE et al. Evaluación del estado de conservación de los bosques montanos en los Andes tropicales. Ecosistemas 2012; 21: 148-166.

23. Cascante A. Guía para la recolecta y preparación de muestras botánicas. Herbario Nacional (CR), Museo Nacional de Costa Rica, San José, Costa Rica. 2008; : 10.

24. Andrade JM, Lucero H, Armijos C. Ethnobotany of Indigenous Saraguros: Medicinal Plants Used by Community Healers 'Hampiyachakkuna' in the San Lucas Parish, Southern Ecuador. BioMed Research International .2017.

25. Tinitana Imaicela F. Composición florística y etnobotánica de las diferentes formaciones vegetales de la provincia de Loja, Ecuador. 2014.

26. Tene V, Malagón O, Finzi P, Vidari G, Armijos C, Zaragoza T. An ethnobotanical survey of medicinal plants used in Loja and Zamora-Chinchipe, Ecuador. Journal of Ethnopharmacology 2007; 111: 63-81.

27. MissouriBotanicalGardenTropicosDatabase. Tropicos Home. 2020.https://www.tropicos.org.

28. Jørgensen PM, León-Yánez S (eds.). Catálogo de las plantas vasculares del Ecuador. Missouri Botanical Garden, 1999.
29. CatálogodelaVida. Catálogo de la vida Home Page. 2020.https:// www.catalogueoflife.org.

30.Brito J, Camacho MA, Romero V, Vallejo A. BioWeb. Mamíferos del Ecuador. Pontificia Universidad Católica del Ecuador. 2019. https://bioweb.bio/faunaweb/mammaliaweb.

31. GlobalBiodiversityInformationFacility. GBIF Home Page. 2020. https://www.gbif.org.

32. UICN. The IUCN Red List of Threatened Species. Version 2020-1. 2020.https://www.iucnredlist.org.

33. León-Yánez S, Valencia R, Pitman N, Endara L, Ulloa Ulloa C, Navarrete $\mathrm{H}$. Libro rojo de las plantas endémicas del Ecuador. 2nd ed. Publicaciones del Herbario QCA, Pontificia Universidad Católica del Ecuador: Quito, 2011.

34. Tirira DG. Libro Rojo de los mamíferos del Ecuador. 2nd ed. Fundación Mamíferos y Conservación, Pontificia Universidad Católica del Ecuador y Ministerio del Ambiente del Ecuador: Quito-Ecuador, 2011.

35. Tirira DG. Guía de campo de los mamíferos del Ecuador. 2nd ed. Asociación Ecuatoriana de Mastozoología y Editorial Murciélago Blanco: Quito, 2017.

36. Tirira DG, Brito J, Burneo SF, AEM C de D de la. Mamíferos del Ecuador: Lista actualizada de especies. Versión 2020.1. 2020.

37. Trotter RT, Logan MH. Informant Consensus: A New Approach for Identifying Potentially Effective Medicinal Plants. In: Plants in Indigenous Medicine and Diet. Bedfore Hills: New York, 1986, pp 91-112.

38. Heinrich M, Ankli A, Frei B, Weimann C, Sticher O. Medicinal plants in Mexico: Healers' consensus and cultural importance. Social Science \& Medicine 1998; 47: 1859-1871.

39.Phillips OL. Some quantitative methods for analyzing ethnobotanical knowledge. In: Alexiades M. (ed). Selected Guidelines for Ethnobotanical Research. A Field Manual. The New York Botanical Garden: Nueva York, United States, 1996, pp 172-197.

40.Germonsén-Robineau L. Hacia una farmacopea Caribeña. Edición TRAMIL 7: Investigación Científica y Uso Popular de Plantas Medicinales en el Caribe. Université des Antilles, Universidad de Antioquía: Santo Domingo, 1995.

41. Friedman J, Yaniv Z, Dafni A, Palewitch D. A preliminary classification of the healing potential of medicinal plants, based on a rational analysis of an ethnopharmacological field survey among Bedouins in the Negev Desert, Israel. Journal of Ethnopharmacology 1986; 16: 275-287.

42. Añazco M. Productos Forestales No Madereros (PFNM) en el Ecuador.. una aproximación a su diversidad y usos. Lyonia 2006; 10: 137-144.

43. Aguirre Z, Rivera M, Granda V. Productos forestales no maderables de los bosques secos de Zapotillo, Loja, Ecuador. Arnaldoa 2019; 26: 575-594

44.Pineda C, Jumbo N. Fernandez P. Jaramillo N. Productos Forestales No Maderables De Origen Vegetal En Cinco Comunidades De La Parroquia Manú, Saraguro, Provincia De Loja. Bosques Latitud Cero 2019; 9: 46-57.

45. Aguirre Z, Betancourt Y, Geada G. Productos forestales no maderables de los bosques secos del cantón Macará, Loja-Ecuador. Revista forestal Baracoa 2012; 31: 1-9.

46. Rios M, Tinitana F, Jarrín- $v$, Donoso N, Romero-Benavides JC. 'Horchata' drink in Southern Ecuador: Medicinal plants and people's wellbeing. Journal of Ethnobiology and Ethnomedicine 2017; 13. doi:10.1186/s13002-017-0145-z.

47. Bussmann RW, Sharon D. Traditional medicinal plant use in Loja province, Southern Ecuador. Journal of Ethnobiology and Ethnomedicine 2006; 2: 1-11.

48. de laTorre L, Alarcón S. D, Kvist LP, Salazar Lecaro J. Usos medicinales de las plantas. In: de laTorre L, Navarrete $H_{\text {, Muriel }} P_{\text {, }}$ Macía MJ, Balslev H (eds). Enciclopedia de las Plantas Útiles del Ecuador. Herbario QCA de la Escuela de Ciencias Biológicas de la Pontificia Universidad Católica del Ecuador \& Herbario AAU del Departamento de Ciencias Biológicas de la Universidad de Aarhus, 2008, pp 105-114.

49. Rivero-Guerra AO. Diversidad y distribución de los endemismos de Asteraceae (Compositae) en la Flora del Ecuador. Collectanea Botanica 2020; 39: 46 
50.Panero JL, Funk VA. The value of sampling anomalous taxa in phylogenetic studies: Major clades of the Asteraceae revealed. Molecular Phylogenetics and Evolution 2008; 47: 757-782.

51. Gallego Aristizabal JH. Plantas competidoras de cultivos de uso medicinal. In: Rios M, Borgtoft Pedersen H (eds). Las Plantas y el Hombre. Memorias del Primer Simposio Ecuatoriano de Etnobotánica y Botánica Económica. Abya-Yala: Quito -Ecuador, 1991, pp 223-228.

52. Minga S, Díaz N, Mendoza Z. Productos forestales no maderables de origen vegetal de cinco comunidades del cantón Yacuambi, Zamora Chinchipe. Bosques Latitud Cero 2017; 7: 72-89.

53. de la Torre L, Navarrete H, Muriel P, Macía MJ, Balslev H. Enciclopedia de las plantas Útiles en Ecuador. Primera. Herbario QCA de la Escuela de Ciencias Biológicas de la Pontificia Universidad Católica del Ecuador \& Herbario AAU del Departamento de Ciencias Biológicas de la Universidad de Aarhus: Quito -Ecuador, 2008.

54.Aguirre-Mendoza Z, Yaguana Puglla C, Merino B. Plantas medicinales de la zona andina de la provincia de Loja. 1st ed. Herbario y Jardín Botánico" Reinaldo Espinosa": Loja-Ecuador, 2014

55. Jácome I, Tanchima V, Santi P, Vargas C. Etnozoología quichua para la conservación de los mamíferos ungulados en la Amazonía central del Ecuador provincia Pastaza. Revista Amazónica Ciencia y Tecnología 2013; 2: 172-185.

56. Jiménez A, Pincay F, Ramos M, Mero O, Cabrera C. Utilización de productos forestales no madereros por pobladores que conviven en el bosque seco tropical. Revista Cubana de Ciencias Forestales: CFORES 2017; 5: 270-286.

57. Solano Rivera JM. Análisis cuantitativo del conocimiento tradicional sobre plantas utilizadas para el tratamiento de enfermedades antitumorales y antiinflamatorias en las parroquias rurales del cantón Macará de la provincia de Loja. 2016.

58. Tinitana F, Rios M, Romero J, de la Cruz Rot M, Pardo-de-Santayana M. Medicinal plants sold at traditional markets in southern Ecuador. Journal of Ethnobiology and Ethnomedicine 2016; 12.

59.Stepp JR. The role of weeds as sources of pharmaceuticals. Journal of Ethnopharmacology 2004; 92: 163-166.

60.Nuñez MA, Simberloff D. Invasive species and the cultural keystone species concept. Ecology and Society 2005; 10: 4-7.

61. Hart G, Gaoue OG, De La Torre L, Navarrete H, Muriel P, Macía MJ et al. Availability, diversification and versatility explain human selection of introduced plants in Ecuadorian traditional medicine. PLoS ONE 2017; 12: 1-16.

62. UICN. Categorías y Criterios de la Lista Roja de la UICN: Version 3.1. 2nd ed. Unión Internacional para la Conservación de la Naturaleza y de los Recursos Naturales (UICN): Gland, Suiza, 2012.

63. Alves RR da N. Zooterapia: importancia, usos e implicaciones conservacionistas. In: Costa Neto EM, Santos Fita D, Vargas Clavijo M (eds). Manual de Etnozoología. Una guía teórico-práctica para investigar la interconexión del ser humano con los animales. Tundra Ediciones: Valencia-España, 2009, pp 165-175.

64. Roberson E. Medicinal plants at risk. A native plant conservation campaign report. Center for Biological Diversity, 2008.

65. Tandon V. The Risks of the loss of Medicinal Plants for Livelihood and Health Security in South Asia. In: Miththapala S (ed). Conserving Medicinal Species: Securing a healthy future. IUCN: Ecosystems an Livelihoods Group, Asia: Asia, 2006, pp 32-39.

66. Añazco Romero MJ. Productos Forestales no Madereros (PFNM) Un Aporte al Manejo Forestal Sostenible. In: Grijalva Olmedo J, Ramos Veintimilla R, Vera Vélez R, Barrera Aguilar P, Sigcha Morales $F$ (eds). Primer Encuentro Nacional de Bosques, Recursos Genéticos Forestales y Agroforestería. Memorias del Evento. Instituto Nacional Autónomo de Investigaciones Agropecuarias. Quito -Ecuador, 2013, pp 25-29.

67. Yánez P. Un vistazo a la conservación biológica en Ecuador. Qualitas 2014; 7: 80-84

68. Lavariega MC, Martín-Regalado N, Monroy-Gamboa AG, Briones-Salas M. Estado de conservación de los vertebrados terrestres de Oaxaca, México. Ecosistemas y Recursos Agropecuarios 2017; 4: 135-146.

69. Martínez de la Vega G. Resumen ejecutivo. Factores de presión. In: Martínez de la Vega G, Flores J (eds). La biodiversidad en San Luis Potosí. Estudio de Estado. Comisión Nacional para el Conocimiento y Uso de la Biodiversidad (CONABIO): Ciudad de México, 2019, pp 115-117.
70.García-Olivares V, Zaragoza-Trello C, Ramirez J, Guerrero-Peñaranda A, Ruiz C. Caracterización rápida de la biodiversidad usando morfometría geométrica: Caso de estudio con abejas sin aguijón (Apidae: Meliponini) del sur de Ecuador. ACl Avances en Ciencias e Ingenierías 2015; 7: B32-B38.

71. Angulo A, Rosero R, González M. Estudio etnobotánico de las plantas medicinales utilizadas por los habitantes del corregimiento de Genoy, Municipio de Pasto, Colombia. Universidad y Salud 2012; 14: 168-185.

72. de la Rosa P, Vásquez M, Villegas Y, Jerez M. Los huertos familiares y la seguridad alimentaria de Cuilapam de Guerrero, Oaxaca, México. Revista Mexicana de Agroecosistemas 2014; 1: 40-51.

73. Nasi R, Brown D, Wilkie D, Bennett E, Tutin C, van Tol G et al. Conservación y utilización de recursos provenientes de la vida silvestre: la crisis de la carne de caza. Serie técn. Secretaría del Convenio sobre la Diversidad Biológica, Montreal y Centro para la Investigación Forestal Internacional (CIFOR), Bogor, 2008.

74. Tirira DG. Uso de la fauna por el pueblo Waorani, Amazonía del Ecuador. In: Tirira DG, Rios M (eds). Monitoreo Biológico Yasuní. Volumen 8: Uso de flora y fauna por el pueblo Waorani, Amazonía del Ecuador. Ecuambiente Consulting Group: Quito-Ecuador, 2018, p 316.

75. Camacho Martinez AV. El uso medicinal de la fauna silvestre y sus implicaciones para la conservación en el municipio del valle del Guamuez, Putumayo, Colombia. 2013.

76. Macias D, Ramírez B, Ramírez H, Varona G. Productos forestales no maderables del área costera del departamento del Cauca. In: Sanabria O (ed). Valoración del conocimiento, uso, manejo y prácticas de conservación de la diversidad de recursos forestales no maderables en diferentes ambientes socioculturales de la región del Pacífico colombiano. Asociación Colombiana de Botánica. Samava Impresiones, 2013, pp 47-78.

77. Lorenzo C, Cruz L, Naranjo E, Barragán F. Uso y Conservación de Mamíferos Silvestres en una Comunidad de las Cañadas de la Selva Lacandona, Chiapas, México. Etnobiología 2007; 5: 99-107.

78. Estrada D, Rosas O, Parra F, Guerrero J, Tarango L. Valor de uso, importancia cultural y percepciones sobre mamíferos silvestres medianos y grandes en la Mixteca Poblana. Acta Zoológica Mexicana (NS) 2018; 34: 1-15.

79. García A, Valle R, Monroy R. Aprovechamiento tradicional de mamíferos silvestres en Pitzotlan, Morelos, México. Revista Colombiana de Ciencia Animal - RECIA 2018; 10: 111-123.

80.Zavala Z, Segura H, Ávila DM, Herrera N, Catalán E, Sarabia G. Valoración cultural y uso de la fauna silvestre en San Vicente de Benítez, Guerrero, México. Revista Etnobiología 2018; 16: 78-92.

81. Lavandera Rodríguez I. Curación de heridas sépticas con miel de abejas. RevCubana de Cirugía 2011; 50: 187-196.

82. Schencke C, Vásquez B, Sandoval C, del Sol M. El rol de la miel en los procesos morfofisiológicos de reparación de heridas. International Journal of Morphology 2016; 34: 385-395.

83. Cueva E. Árboles y arbustos productores de frutos comestibles en la provincia de Loja. In: Rios M, Pedersen H (eds). Uso y manejo de recursos vegetales. Memorias del Segundo Simposio Ecuatoriano de Etnobotánica y Botánica Económica. Quito -Ecuador, 1997, pp 223-233.

84. Van den Eynden V, Cueva E, Cabrera O. Plantas silvestres comestibles del sur del Ecuador-Wild edible plants of sourthern Ecuador. Abya-Yala: Quito-Ecuador, 1999

85. Van den Eynden V, Cueva E, Cabrera O. Wild Foods from Southern Ecuador. Economic Botany 2003; 57: 576-603.

86. Jima TT, Megersa M. Ethnobotanical Study of Medicinal Plants Used to Treat Human Diseases in Berbere District, Bale Zone of Oromia Regional State, South East Ethiopia. Evidence-based Complementary and Alternative Medicine 2018; 2018: 16.

87. Mata Pinzón S, Pérez Ortega G, Reyes Chilpa R. Plantas medicinales para el tratamiento del Susto y mal de ojo. Análisis de sus posibles efectos sobre el sistema nervioso central por vía transdérmica e inhalatoria. Revista Etnobiología 2018; 16: 30-47.

88. Echeverria G, Graell J, López L, Lara I. La calidad organoléptica de la fruta. Horticultura Internacional 2008; 61: 26-36.

89. Vargas Ortiz MA, de la Cruz Medina J, Oliart Ros RM, Rebolledo Martínez A, Ramírez JA, García HS. Efecto de las altas presiones hidrostáticas sobre los cambios poscosecha de mango (Mangifera indica) 'manila' en madurez fisiológica. CienciaUAT 2013; 8: 52-63. 
90.García Mogollón C, Cury Regino KI, Dussán Sarria S. Comportamiento Poscosecha y Evaluación de Calidad de Fruta Fresca de Guayaba en Diferentes Condiciones de Almacenamiento. Revista Facultad Nacional de Agronomía 2011; 64: 6207-6212.

91. Gimeno E. Frutos secos y salud. Offarm: farmacia y sociedad 2002; 21: 90-96.

92. López León R, Ureña Solís J. Propiedades antioxidantes de los frutos secos y la disminución del colesterol total y LDL- colesterol. Revista Costarricense de Salud Pública 2012; 21: 87-91.

93. Lewis G, Klitgaard BB. Leguminosas del sur de Ecuador. In: Aguirre-Mendoza Z, Madsen J., Cotton E, Balslev H (eds). Botánica Austroecuatoriana - Estudios sobre los recursos vegetales en las provincias de El Oro, Loja y Zamora-Chinchipe. Abya-Yala: Quito-Ecuador, 2002, pp 185-224

94. Mezzatesta P, Raimondo E, Flores DG, Llaver C. Contenido de boldina en tisanas y sus implicancias en salud. Diaeta 2016; 34: 19-24.

95. López Luengo MT. Formas de administración más habituales de plantas medicinales. Offarm: farmacia y sociedad 2002; 21: 122-125.

96.Villegas-Novoa C, Moreno-Jiménez MR, Rocha-Guzmán NE. Infusión de la planta medicinal Buddleja scordioides Kunth utilizada para tratar la inflamación intestinal. CienciaUAT 2020; 14: 21-33.

97. García Bacallao L, Rojo Dominguez DM, García Gomez LV, Hernández Angel M. Plantas con propiedades antiinflamatorias. Revista Cubana de Investigaciones Biomedicas 2002; 21: 214-216.

98.Paniagua-Zambrana NY, Bussmann RW, Romero C. Juglans boLiviana (C. DC.) Dode Juglans neotropica Diels Juglandaceae. In: Paniagua-Zambrana NY, Bussmann RW (eds). Ethnobotany of the Andes. Springer International Publishing: Cham, Switzerland 2020, pp 1-9.

99.Paredes DJ, Buenaño-Allauca MP, Mancera-Rodríguez NJ. Usos de plantas medicinales en la comunidad San Jacinto del cantón Ventanas, Los Ríos-Ecuador. Revista UDCA Actualidad \& Divulgación Científica 2015; 18: 39-50.

100. Angulo A, Mosquera L. Patologías Andinas. In: Orellana A, Achig D, Angulo A, Barrera G, Brito L, Mosquera L (eds). Sabiduría Ancestral Andina y Uso de Plantas Medicinales. Principios y Prácticas de la Medicina Tradicional en Ecuador. Talleres Gráficos de la Universidad de Cuenca: Cuenca-Ecuador, 2020, pp 27-43.

101. Paniagua-Zambrana NY, Bussmann RW, Romero C. Ruta chalepensis L. Ruta graveolens L. Rutaceae. In: Paniagua-Zambrana NY, Bussmann RW (eds). Ethnobotany of the Andes. Springer International Publishing: Cham, Switzerland, 2020, pp 1-9.

102. López García B, Ortonobes Roig S, García Rebollar C. Ungüentos, pomadas, cremas, geles y pastas: ¿es todo lo mismo? Formación Activa en Pediatría de Atención Primaria 2015; 8: 183-187.

103. Armijos C, Cota I, González S. Traditional medicine applied by the Saraguro yachakkuna: A preliminary approach to the use of sacred and psychoactive plant species in the southern region of Ecuador. Journal of Ethnobiology and Ethnomedicine 2014; 10: $1-26$.

104. Cavender AP, Albán M. The use of magical plants by curanderos in the Ecuador highlands. Journal of Ethnobiology and Ethnomedicine 2009; 5: 1-9.

105. Marinoff MA, Martínez JL, Urbina MA. Precauciones en el empleo de plantas medicinales. Boletín Latinoamericano y del Caribe de Plantas Medicinales y Aromáticas 2009; 8: 184-187.

106. Ladio A, Lozada M. Nontimber Forest Product use in two human populations from Northwest Patagonia: A quantitative approach. Human Ecology 2001; 29: 367-380.

107. Rowe AP. Natural Dyeing Techniques. In: Rowe AP, Miller LM, Meisch LA (eds). Weaving and Dyeing in Highland Ecuador. University of Texas Press: United States of America, 2007, pp 251-277.

108. Abalos-Romero M. Productos Forestales no Madereros de Ecuador. In: Proyecto Informacion y análisis para el manejo forestal sostenible. Santiago de Chile, 2001, pp 97-106.

109. Albán-Castillo J, Espinoza G, Rojas R, Díaz Santibáñez C. El color en la memoria: tintes vegetales usados en la tradición de las comunidades andinas y amazónicas peruanas. Ecología Aplicada 2018; 17: 85-96.
110. de La Torre L, Cummins I, Logan E. Agave americana and Furcraea andina: Key Species to Andean Cultures in Ecuador. Botanical Sciences 2018; 96: 246-266.

111. Carrión Paladines HV. El etnoecosistema en la producción de plantas medicinales en la comunidad 'El Carmelo', parroquia Chuquiribamba, Loja, Ecuador. 2012.

112. Guapucal Cuasanchir M, Benavides C. XS, Sinisterra KX Valoración participativa del uso y manejo del huerto mixto. Revista de Ciencias Agrícolas 2019; 36: 46-58.

113. Hillbrand A, Borelli S, Conigliaro M, Olivier A. Agroforesteria para la restauración del paisaje: explorando el potencial de la agroforesteria para mejorar la sostenibilidad y la resiliencia de los paisajes degradados. Roma, 2017.

114. McNeely JA. The Risks from the Loss of Medicinal Species. In: Miththapala S (ed). Conserving Medicinal Species: Securing a healthy future. IUCN: Ecosystems an Livelihoods Group, Asia: Asia, 2006, pp 17-31.

115. León Merino A, Rivera Peña R, Hernández Juárez M, Sangerman Jarquín DM, Jiménez Sánchez L, Valtierra Pacheco E. Aprovechamiento de productos forestales no maderables en la comunidad Pensamiento Liberal Mexicano, Oaxaca. Revista Mexicana de Ciencias Agrícolas 2017; 18: 3725-3738.

116. Bennett EL, Robinson JG. The Sustainability of Hunting in Tropical Forests. In: Bennett EL, Robinson JG (eds). Hunting of Wildlife in Tropical Forests: Implications for Biodiversity and Forest Peoples. The International Bank for Reconstruction: Washington, D.C, 2000, pp 5-10.

117. Vickers W. Hunting Yields and Game Composition over Ten Years in an Amazon Indian Territory. In: Robinson JG, Redford K. (eds). Neotropical Wildlife Use and Conservation. University of Chicago Press: Chicago and London, 1991

118. Watson JEM, Evans T, Venter O, Williams B, Tulloch A, Stewart $\mathrm{C}$ et al. The exceptional value of intact forest ecosystems. Nature Ecology and Evolution 2018; 2: 599-610.

119. Tirira DG, Rios M. Monitoreo Biológico Yasuní. Volumen 8: Uso de la flora y fauna por el pueblo Waorani, Amazonía del Ecuador. 1st ed. Ecuambiente Consulting Group: Quito-Ecuador, 2018.

120. Briceño Fonseca LM, Mahecha Garzón AG, Triana Gómez MA. Recuperación etnobotánica del uso tradicional no maderable del bosque secundario en el municipio de Nocaima, Cundinamarca. Revista Mutis 2017: 7: 48-66.

121. de Albuquerque UP. Re-examining hypotheses concerning the use and knowledge of medicinal plants: A study in the Caatinga vegetation of NE Brazil. Journal of Ethnobiology and Ethnomedicine 2006; 2: 10.

122. Jaramillo MA, Castro M, Ruiz-Zapata T, Lastres M, Torrecilla P, Lapp M et al. Estudio etnobotánico de plantas medicinales en la comunidad campesina de Pelelojo, municipio Urdaneta, estado Aragua, Venezuela. Ernstia 2014; 24: 85-110.

123. Ramos TI, Castro KA, Escalante LS, Vispo NS. Advances in Clinical Research in Ecuador. Therapeutic Innovation and Regulatory Science 2016; 51: 307-313.

124. Ullah S, Muhammad N. Evaluation of Informant Consensus Factor of Medicinal Plants Used in Shamozai Valley SWAT, KPK, Pakistan. International Journal of Medicinal Plants and Natural Products 2018; 4: 1-7.

125. Kvist LP, Aguirre Z, Sánchez O. Bosques montanos bajos occidentales en Ecuador y sus plantas útiles. In: Morales R. M, Øllgaard B, Kvist LP, Borchsenius F, Balslev H (eds). Botánica Económica de los Andes Centrales. Universidad Mayor de San Andrés: La Paz-Bolivia, 2006, pp 205-223.

126. Mora Roman G, Quizhpe Coronel W, Castro P, Jadán O. Conocimiento sobre productos forestales no maderables en dos pisos florísticos: piemontano y montano bajo, en bosque semi-caducifolio Santa Rufina, Loja, Ecuador. Bsoques Latitud Cero 2017; 7: 39-54.

127. Marín-Corba C, Cárdenas-López D, Suárez-Suárez S. Utilidad del valor de uso en etnobotánica. Estudio en el departamento de Putumayo (Colombia). Caldasia 2005; 27: 89-101.

128. Galeano G. Forest use at the Pacific Coast of Choco, Colombia: A quantitative approach. Economic Botany 2000; 54: 358-376. 
129. Bennett BC, Prance GT. Introduced plants in the indigenous pharmacopoeia of northern South America. Economic Botany 2000; 54: 90-102.

130. Begossi A, Hanazaki N, Tamashiro JY. Medicinal plants in the Atlantic Forest (Brazil): knowledge, use, and conservation. Human Ecology 2002; 30: 281-299.

131. Fernández-Cusimamani E, Espinel-Jara V, Gordillo-Alarcón S, Castillo-Andrade R, Ziarovska J, Zepeda-Del Valle JM et al. Estudio etnobotánico de plantas medicinales utilizadas en tres cantones de la provincia Imbabura, Ecuador. Agrociencia 2019; 53: 797-810.

132. Cantos Zambrano JA. El uso de las especies medicinales como productos forestales no maderables en el sitio la Tranca Arriba, parroquia San Plácido. 2019.

133. Zambrano-Intriago LF, Buenaño-Allauca MP, Mancera-Rodríguez NJ, Jiménez-Romero E. Estudio etnobotánico de plantas medicinales utilizadas por los habitantes del área rural de la Parroquia San Carlos, Quevedo, Ecuador. Revista Universidad y Salud 2015; 17: 97-111.

134. Jiménez-Romero EM, Moreno-Vera AN, Villacís-Calderón AC, Rosado-Sabando JK, Morales Moreira DM, Bravo Bravo AD. Estudio etnobotánico y comercialización de plantas medicinales del bosque protector Murocomba y su área de influencia del cantón Valencia, Ecuador. Ciencia y Tecnologia Agropecuaria 2019; 20: 491-506.
135. Aguirre Z, Cueva E, Merino B, Quizhpe W, Valverde A. Evaluación ecológica rápida de la vegetación en los bosqes secos de La Ceiba y Cordillera Arañitas, provincia de Loja, Ecuador. In: Vázquez M., Larrea M, Suárez L, Ojeda P (eds). Biodiversidad en los bosques secos del suroccidente de la provincia de Loja: un repote de las evaluaciones ecológicas y socioeconómicas rápidas. Ecociencia, Ministerio del Ambiente, Herbario Loja y Proyecto Bosque Seco: Quito, 2001, pp 1535

136. Sánchez O, Kvist LP, Aguirre Z. Bosques secos en Ecuador y sus plantas útiles. In: Morales R. M, Øllgaard B, Kvist LP, Borchsenius $\mathrm{F}$, Balslev H (eds). Botánica Económica de los Andes Centrales. Universidad Mayor de San Andrés: La Paz-Bolivia, 2006, pp 188-204.

Received: 16 Julio 2021

Accepted: 10 Septiembre 2021 